\title{
The Application of the Defects of VR in Education
}

\author{
Ren Zhiwei ${ }^{1, *}$ \\ ${ }^{1}$ Department of Journalism, Nanjing University of Aeronautics and Astronautics, Jiangning, Nanjing, Jiangsu, China \\ *vzhiwei@gmail.com
}

\begin{abstract}
VR technology has significant advantages such as interactivity and imagination, but also has significant defects such as "motion sickness" and space limitation. This article aims to explore the application of VR technology's defects in the field of education through analysis of the defects of VR technology, so as to provide future researchers with reference to the direction of technology research and development, which has certain theoretical and practical significance.
\end{abstract}

Keywords: VR technology; VR defects; disaster education; education model

\section{VR 技术的缺陷在教育领域的运用探索}

\author{
任知微 ${ }^{1,}$ \\ 南京航空航天大学新闻系, 江宁, 南京, 江苏, 中国 \\ vzhiwei@gmail.com

\section{摘要} \\ VR 技术具有交互性、构想性等显著优势，同时也具有 “晕动症”、空间限制等显著缺陷。本文旨在通过 \\ 对 VR 技术的缺陷分析, 探索 VR 技术的缺陷在教育领域的应用可能, 以此为今后的研究者提供技术研 \\ 发的方向参考, 具有一定的理论与现实意义。
}

关键词: VR 技术; VR 缺陷; 灾难教育; 教育模式

\section{1. 前言}

近年来, VR 技术的发展势头愈加强劲, 应用范围愈 加广泛, 特别在 3D 仿真模拟上, 能够高度真实地还原 现场, 给受众带来 “沉浸式” 体验, 具有独特优势。目 前, VR 技术的应用主要集中于建筑、游戏、医疗、教育、 军事和体育领域。其中, 教育、游戏和体育领域的应用 主要面向大众, 其经济前景广阔, 社会价值显著。VR 技 术在教育领域的应用已有不少成果, 早在 2016 年, NASA 就与 Play Station VR 等平台进行合作, 推出了 Mission: ISS 等多个面向公众的 APP, 向大众普及航空 航天知识, 取得了不俗的效果。除了科普教育, VR 技术 在教育领域还有很大的应用缺口等待学者和研发者填 补。

根据 IDC 发布的《2019 年中国智能终端市场十大预 测》, 2019 年会有 $20 \%$ 的教育用户考虑 VR 技术; 2020 年则会有 50\%的用户尝试运用 VR 或者 AR 产品。2015 年, 中国国务院印发《“十三五”国家战略性新兴产业发 展规划》, 其中提到要 “推动数字创意在教育领域的应 用, 提升学习内容创意水平, 加强数字文化教育产品开 发和公共信息资源深度利用, 推动教育服务创意化。” VR 技术作为数字创意的重要一环, 备受中国政府重视。但
是, VR 技术目前还存在很多缺陷, 短期内很难做出突破 性改观。这时就需要转变思路, 化 “缺陷” 为 “优势”, 让 VR 技术的缺陷也能尽其所能地发挥用处。

\section{VR 技术的缺陷特性}

VR 技术的具体缺陷是 VR 技术的衍生特性。同时， 作为衍生特性的具体缺陷也有其本身所具备的内部特 性。对这些具体缺陷的内部特性进行分析研究, 是化“缺 陷” 为 “优势” 的必要前提。



图 1 特性分析示意图 


\section{1. 晕动症的特性}

晕动症的产生源于人脑的认知错位。当人们的实际 运动与虚拟世界里的运动不相符合之时, 即会产生 “晕 眩感”, 这是军动症的具体表现, 同时也是军动症的最显 著特性。此外, VR 设备的高延时也是引发晕动症的一大 原因。作为缺陷特性的晕眩感会给用户带来许多不适的 症状, 包括头晕、恶心、呕吐、头痛和唾液分泌增多等。

${ }^{[1]}$ 晕动症作为 VR 技术最显著的负面效果之一, 严重影 响用户在运动位移时所产生的距离感和方向感, 从而导 致身体失衡, 以致无法精确地操纵虚拟空间。身体失衡 对体育类 VR 产品的使用效果影响显著, 失控感则对 VR 游戏的交互效果影响显著。有研究表明, 保持坐姿或站 姿的稳定, 能够在晕动症严重的情况下缩短晕眩时长。 并且, 用户的个体差异对晕动症的敏感程度也具有显著 差异。 ${ }^{[2]}$ 除了晕眩感, 纱门效应是晕动症的另一特性。 所谓纱门效应, 即像素不足产生空隙, 从而带来线条舞 动和分离式闪伢现象, 在感官上接近于 “模糊感”。晕眩 感和模糊感相互影响、相互渗透, 是 VR 产品研发工作 的重大难题。

\section{2. 空间限制的特性}

空间限制是 VR 产品的另一大缺陷。一方面, VR 技 术可以具化用户的想象空间。例如在医学仪器的使用中, VR 技术可以将人体的各个组织和器官进行具象呈现, 在 医学教育和医学实践方面起到了化 “平面”为 “立体” 的作用。最为典型的运用包括 Mentie 公司研制的 Proeedieus MIST 系统和 Surgieal Seience 研制的 Lapsim 系统等。它们都体现了 VR 技术在虚拟空间的延 展效能。但是另一方面, 人体在使用 VR 设备之后也会 受到实际空间的限制。从安全角度看, 处在交互运动中 的使用者会受到较为严格的空间运动限制, 原因主要有 两点。其一, 人类的两足直立是一种天然的不平衡状态。 用户在进入虚拟视像之后, 会严重影响其对现实空间的 距离感知和方向感知, 从而产生安全隐患。其二, VR 眼 镜或头盔的自重通常在 300 克以上, 这无疑会给头部造 成重量负担, 影响人体的运动平衡。因此, 用户在使用 VR 设备的过程中, 身体位移往往被加以严格的限制, 这 就导致人体在运动交互的过程中必然受阻。

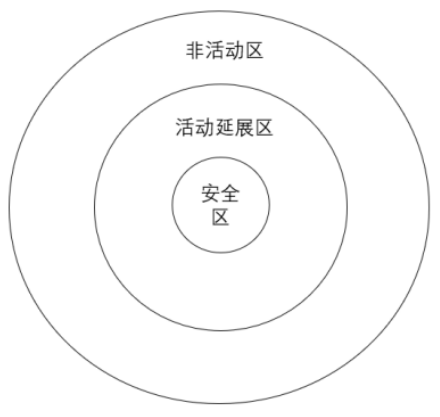

图 2 运动范围示意图

\section{VR 技术的缺陷与灾难教育的结合}

通过对 VR 技术缺陷的分析, 本文发现, 这些缺陷 本身可以被加以利用, 使之实现价值转嫁。晕眩感是晕 动症的最显著特征, 而在以地震为主要类型的自然灾害 中, 晕眩感恰好也是受灾者必将产生的生理反应。因此, 晕眩感可以和部分灾难教育加以结合。运用 VR 技术对 受教育者进行灾难知识的普及和受灾经验的传递, 是化 “缺陷”为 “优势” 的重要方案之一。目前, VR 系统主 要有一下几种分类: 桌面 VR 系统、沉浸式 VR 系统、遥 现 VR 系统和分布式 VR 系统。教育领域常用的是沉浸式 VR 系统。沉浸式系统是实现 VR 技术缺陷运用的最佳场 景。

\section{1. 灾难教育的扩展}

杨挺在《灾难教育: 学校教育的重要课题——写在 汶川地震灾难之后》中将灾难教育分为“灾难知识教育” “灾难应对教育” “灾难心理教育”和 “灾难体验教育” 四大类型。 ${ }^{[3]}$ 其中, 灾难心理教育特指灾难期间的心理 抗压能力训练。灾难体验教育也仅限于现实模拟，中国 有许多省份在科技馆内设有 “地震体验馆”。但是随着 VR 技术不断介入教育领域, 灾难心理教育和灾难体验教 育的外延也应得到相应的扩展。

在灾难心理教育方面, 除了必要的抗压能力训练之 外，心理警示功能也必须加以考量。VR 技术是心理警示 的重要手段。在传统的教育模式中, 说教占据重要分量, 但效果甚微。“灾难二分法” 将灾难分为自然灾难和人为 灾难两种，灾难教育中的心理警示作用则主要针对后者。 VR 技术多以全景视频为主要呈现手段, 通过强视觉刺激 使内容直抵受众内心。同时, VR 技术的运用能够为受教 育者创造 “沉浸感” 与 “现场感”, 营造灾难现场的仿真 环境, 让他们第一时间 “抵达” 最真实的灾难现场, 更 深刻地感知灾难面前的生命意义与价值, 从而提高受教 育者的灾难防范意识。

在灾难体验教育方面, 除了现实模拟, 虚拟模拟也 成为可能。VR 技术可以带来胜于理性认知的主观感受与 共情体验。传统的现实模拟环境的仿真程度低, 容易使 受教育者产生 “失真” 感。VR 技术改善了这一不足。

除此之外, 灾难体验教育还应包含“体验他人苦难” 的人文主义教育内容, 而非仅仅局限于自我警示。早在 2015 年, 《纽约时报》就发布了一篇有关叙利亚难民的 VR 灾难报道 “The Displaced” (《流离失所》)。 ${ }^{[4]} \mathrm{VR}$ 影片可以使人们了解并感知到远方正在发生的苦难, 并 予以共情。

\section{3. $2 . V R$ 与地震教育的结合}

目前, 火灾和气象灾害的数字防疫技术都有大量研 究, 但关于地震的仿真模拟技术却很少有研究涉猎。地 震方针模拟的模型巨大、数据海量, 普通的二维信息不 
能满足地震仿真模拟的技术需要。GIS、VR、CAD 等技术 成为最关键的技术支撑。 ${ }^{[5]}$ 目前已有 ERT-VR 地震救援 仿真系统和城市地震仿真系统的相关研究, 但是在 VR 产 品方面, 以地震为主体的 VR 影片仍旧存在缺口。特别 是在 VR 技术的“晕眩感”运用方面更是尚无先例。

根据前文所述的灾难教育分类, 以地震教育为主题 的 VR 产品可以被分为“地震知识教育”“地震应对教育” “地震心理教育” 和 “地震体验教育” 四大类型。在中 国国内, 刘永福等人使用 Virtools 等软件开发出远程 防震教育软件，模拟地震发生的不同场景。Ishii Hirotake 等开发了具有建模功能的 VR 地震体验系统。

“地震逃生 PICO 一体机” 的产品也开始出现。这些产 品涵盖了地震知识教育和地震应对教育。但是对于地震 心理教育和地震体验教育的尝试还处于探索阶段。

在地震灾难教育中, “晕眩感”既是受灾者的必然感 受, 同是也是 VR 用户的必然感受, 二者可以自然形成 无缝对接。这一情况使得教育产品的设计者不必再额外 花费成本去营造地震场景的晕眩感。VR 设备本身就能够 带来晕眩感，达到化 “晕眩” 为 “有用” 的正面效果。 与此同时, VR 技术的空间限制特性也严重影响着运动产 品的交互效果。在灾难教育产品中, 其设计更多偏重“沉 浸感知” 而非 “运动交互”。在 “沉浸感知” 中, 空间限 制的缺陷显然对产品效果不具有显著影响, 这又恰好有 效避免了 VR 技术的另一大缺陷对 VR 产品的负面影响。

因而, 仅从 VR 技术的缺陷特性来看, 地震灾难教 育是 VR 产品未来发展的重要方向。

\section{3. VR 灾难教育需进行模式创新}

目前, 虚拟现实技术在灾难教育中的应用虽然发挥 了很大的成效, 但是在教育过程中, 受众处于一种沉浸 的状态, 在这种情况下受众更多的是被动地接受。这是 因为很多 VR 短片都是对场景进行简单的重现, 关于灾 难的形成机制、传播机制都没有进行深入的探讨, 灾难 教育内容不够深入, 覆盖面不广, 没有达到灾难教育的 真正目的。实际上, 灾难教育中 VR 技术的运用应该注 重形式与内容的有机统一。要发挥虚拟现实技术沉浸、 想象和交互的特点, 在展现灾难过程和现场场景上下功 夫, 注重动态呈现, 增强受众自主性、互动性。各大媒 体也可以尝试与 VR 技术公司进行合作, 如新华社对深 圳滑坡事故的 VR 报道中与兰亭数字全景视频制作公司、 财新传媒 VR 队伍等合作制作 VR 新闻, 取长补短, 取得 了不俗的效果。 ${ }^{[6]}$ 此外, 虚拟现实技术在灾难教育中的 应用要扩大地理范围和主题范围, 不直于国内灾难事件, 不停留于场景展示, 加强灾难的深度分析和社会观照。 乔治・华盛顿大学医院发布了一份关于新冠状病毒感染 肺部的 VR 虚拟现实报告, 通过视频可以看到, 在正常 情况下肺部是不透明的, VR 视频中肺部整体被描绘成半 透明的蓝色, 而感染区域则为黄色。除了呈现病毒传播 到整个肺部的原始影响之外, 乔治 - 华盛顿大学的可视 化报告还显示了人体对新冠状病毒的反应, 即创建多个 炎症区, 共同抑制正常的肺功能, 使患者需要使用呼吸
管或呼吸机住院治疗。这一VR 报告, 通过视觉的呈现 效果来震惊读者, 希望通过更为直观的展现, 让人们对 新冠状病毒表现出应有的畏惧。

VR 技术的运用面临 “真实性” 问题，稍不注意就会 对受众带来不真实的第一印象。例如, 2016 年的天津港 爆炸案中，虚拟现实技术的运用夸大了整个受灾程度， 导致受众恐慌情绪蔓延。 ${ }^{[7]}$ 此外，对于灾难现场的刺激 性场景, 可以根据受众的心理承受能力、年龄层次等进 行分析, 引导灾难教育中虚拟现实技术的运用朝着良性、 健康的方向发展。

\section{4. VR 灾难教育应考虑人文关怀}

目前, 关于灾难教育的理解主要局限于将灾难本身 视为一种完全客观的自然现象以及灾难技能教育。在笔 者看来, 灾难的理解是灾难教育的前提, 灾难教育不仅 仅是灾难技能教育, 更应当关注的是面对灾难, 人的心 灵怎样平复的问题。灾难事件的发生往往伴随着经济损 失、人员伤亡，身处其中的受灾群众是痛苦和脆弱的。 如果反复多次会通过虚拟现实技术一次次高度真实地 重现, 受灾群众可能要再次面对可怕的受灾场景。因此, 在灾难教育中, 虚拟现实技术要将重点放在灾难事件本 身上, 力争第一时间真实准确地反映受灾情况, 除了要 客观地展示灾难现场外, 还需要以受众为中心, 如果受 教育者包含受灾群众, 则要设身处地地考虑他们的感受, 同时注重引导人们思考灾难背后的原因。为了避免过于 血腥暴力, 可以通过动画的形式来展现内容, 这样既能 反映灾难事件, 又可以减少对灾区受众的二次伤害。特 别是要展示人们战胜灾难的画面, 例如, 可以利用虚拟 现实技术展示专家团队抗击新型冠状病毒感染肺炎疫 情的场面, 或者是新型冠状病毒感染患者战胜病魔的历 程, 帮助其他患者、家人以及广大社会群体重拾信心。 [8]

\section{VR 技术的专注力教育的结合}

建构主义认为, 学习的本质是一个知识建构的过程。 越来越多的当代学生开始变得有主见、有个性、思维活 跃、有较强的信息素养。所以, 我们的教育者应当给予 这些学生较高的学习决策权, 或帮助他们亲身参与到知 识学习的过程中去。Thomas Davenport 认为, 教学的本 质是一种信息传递，而信息的消耗本质上是对受众注意 力的占有。“注意力的选择性导致想要得到对某事清 晰、完整、深刻的反映, 需要使其心里活动有选择地指 向有关对象”。维系注意力是保障信息传播效果的重要 前提。[9]

VR 产品具有自主感和拥有感, 它们共同决定受教育 者的主导地位。此外, VR 产品的仿真模拟和沉浸式体验, 能够帮助受教育者快速进入专注力集中的状态。“授人 以鱼不如授人以渔”, 一旦我们的 VR 产品可以使受教育 者快速进入学习状态, 成为他们学习前的有效预备, 学 
习成效自然会得到提升。目前为止, 尚未出现专门针对 专注力训练的 VR 产品。根据前文所述, 高延时和空间 位移会引发受教育者在 VR 产品使用过程中的晕动症。 根据这一特点, VR 产品在设计和研发的过程中需要尽可 能地减少时长和运动幅度。在专注力教育中, 二者恰好 都能被有效避免。

\section{5. 结语}

VR 技术具有晕动症和空间限制等缺陷, 这些缺陷可 以与以地震为主的灾难教育进行结合, 同时也可以运用 于专注力教育当中。这些尝试具有一定的创新和现实意 义, 也是未来 VR 产品创新和深化的重要方向。同时, VR 产品的设计也应考虑人文关怀和模式创新。

\section{REFERENCES}

[1] Stanney, K M., Kennedy, R S., Drexler, J M. (1997) Cybersickness is not simulator sickness. Proceedings of the Human Factors and Ergonomics Society annual meeting. USA: SAGE Publications, 41(2): 1138-1142.

[2] Cai, L., Weng, DD., Zhang, ZL., Yu, XY. (2016) The Effect of Virtual-Real Motion Consistency on Virtual Reality Motion Sickness. Journal of System Simulation. 28(09):1950-1956.

[3] Yang, T. (2008) Disaster Education: An Important Subject of School Education-Written after the Wenchuan Earthquake. Chinese Journal of Education, (11):17-20.

[4] Bi, ZY., Li, CR. (2019) The Dilemma and Outlet of VR Reporting in My Country's Disaster News. Young Reporter, (20):17-18.

[5] Chen, XP., Lu, XZ., Z, X., Ren, AZ. (2008) Urban earthquake simulation system based on GIS-CAD-VR. China Civil Engineering Society Computer Application Branch, China Architecture Society Building Structure Branch Computer Application Professional Committee, Zhejiang Provincial Civil Engineering Society. Proceedings of the 14th National Engineering Design Computer Application Academic Conference. China Civil Engineering Society Computer Application Branch, China Architectural Society Building Structure Branch Computer Application Professional Committee, Zhejiang Civil Engineering Society: China Civil Engineering Society.276283.

[6] 36KR, 2015"Visit" Shenzhen Landslide Rescue Site, VR Brings "First Scene" to News. https://36kr.com/p/5041463

[7] Bi, ZY., Li, CR. (2019) The Dilemma and Outlet of VR Reporting in my country's Disaster News. Young Reporter.
(20):17-18.

[8] Jia, QH., Li, C. (2015) Innovation and change of traditional disaster news reports by VR technology. Jin Media, 28(01):25-27.

[9] Chen, SK., Wu, Y. (2020) The advantages and Changes of VR Technology in Eucational Senes: Eperience, visualization and constructivism. hina Media Technology, (04):121-125. 